\title{
The pertinence of jet emitting discs in microquasars. Theory and comparison to observations
}

\author{
Pierre-Olivier Petrucci ${ }^{1}$ Jonathan Ferreira $^{1}$ Gilles Henri $^{1}$ \\ Julien Malzac ${ }^{2}$ and Cedric Foellmi ${ }^{1}$ \\ ${ }^{1}$ Laboratoire d'Astrophysique de GrenOble, \\ Université Joseph Fourier - Grenoble 1 / CNRS \\ UMR 5571, BP 53, 38041 Grenoble Cedex 09, France \\ email: pierre-olivier.petrucci@obs.ujf-grenoble.fr \\ ${ }^{2}$ Centre d'Etude Spatial des Rayonnement \\ UMR 5187 \\ 9, av du Colonel Roche BP 4434631028 Toulouse Cedex 4 France
}

\begin{abstract}
Jet Emitting Disc (JED) has dynamical properties quite different from both the standard and advection dominated discs. It also exhibits three different thermal equilibrium branches at a given radius: two stable (cold and hot) and one intermediate unstable. The hot solution has all the characteristics of the so-called "hot corona" generally invoked in XrB sytems in the Low/Hard states. We detail the energetics and radiative expectations of our model and show their good agreement with those observed in Cygnus X-1 in terms of jet power, jet velocity and spectral emission.
\end{abstract}

Keywords. X-rays: binaries, accretion, accretion disks, magnetic fields: MHD

\section{Introduction}

Jet emitting discs (JED herafter) were originaly studied by Ferreira \& Pelletier (1993) in their magnetized accretion ejection structures model (MAES herafter). This model was developed so as to treat both the accretion disc and the jet it generates consistently. The idea is the same as in earlier studies of magneto-centrifugally launched disc winds (Blandford \& Payne 1982). However, in the MAES, the solution starts from the midplane of the resistive MHD disc and evolves outwards in the ideal MHD wind/jet. This differs drastically from other studies where the disc was only treated as a boundary condition, hence forbidding any precise quantication of the effect of the MHD wind on the disc.

The resolution of the thermal equilibrium of a JED gives three branches, a cold and hot ones, both thermally and viscously stable and an intermediate unstable one. The hot branch, that would be observationally very similar to ADAFs, corresponds to the JED solution discussed in this paper and applied to Cyg. X-1 (Petrucci et al. 2010). Thus JEDs could account for most of the successes of the ADAFs, while explaining jet formation (see Ferreira's talk, this proceeding).

\section{Comparison to Cygnus X-1}

The energetics of the jets and the X-ray corona of Cygnus X-1 have been investigated recently by Malzac et al. (2009 herafter M09). Observations constraint the ratio 

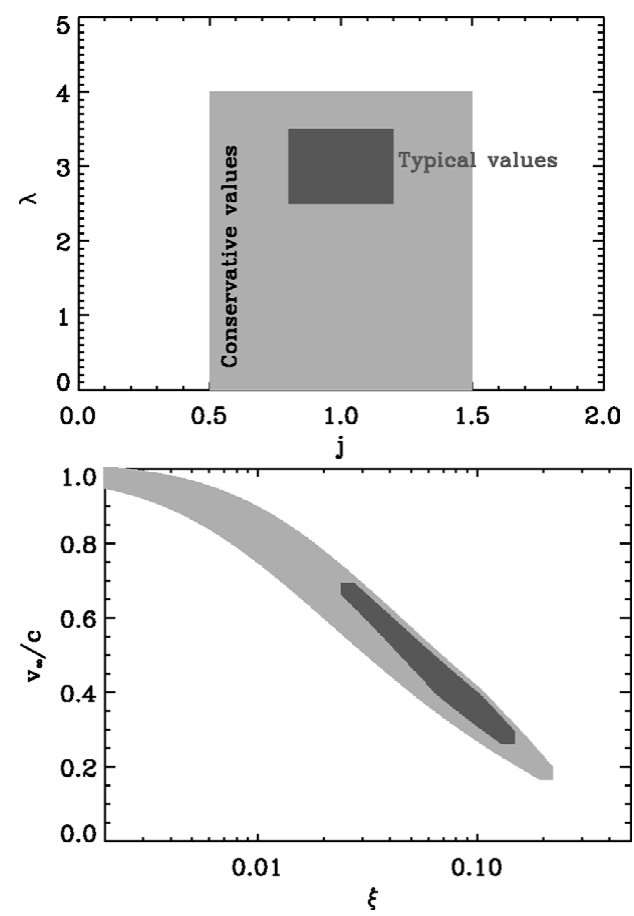

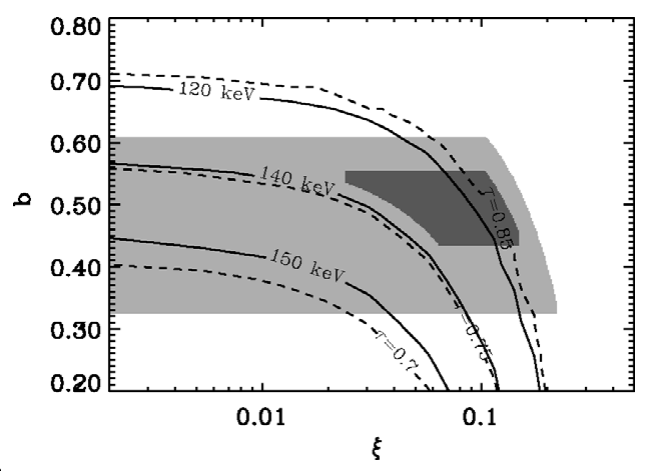

Figure 1. Top-left: the parameter space $j-\lambda$ allowed by the observations. Top right: Observational constraints translated into our JED parameter space $\xi-b$. Overplotted are the contours of temperature (solid lines) and optical depth (dashed lines) for an accretion rate of 1-2\% Edd., typical of Cyg X-1. Bottom-left: terminal jet velocity $V_{\infty} / c$ as function of the disc ejection efficiency $\xi$

$j=P_{j e t s} / L_{h}$ of the total jet kinetic power to the typical X-ray luminosity in the hard state as well as the ratio $\lambda$ of the soft to hard radiative efficiencies to be in the ranges (M09):

$$
0.45 \leqslant j=\frac{P_{j e t s}}{L_{h}} \leqslant 1.5 \text { and } \lambda=\frac{L_{s}}{\dot{M}_{s}} \frac{\dot{M}_{h}}{L_{h}} \leqslant 4
$$

These observational constraints on $j$ and $\lambda$ can be easily translated into constraints on our JED parameters $b$ and $\xi$, where $P_{\text {jets }} \simeq b P_{a c c}$ and $\dot{M}_{a}(R) \propto R^{\xi}$. The top-left panel of Fig. (2) displays the domain in the observed parameter space $j-\lambda$ allowed by the observations. The top right panel shows the same constraints but translated into our JED parameter space $\xi-b$. Overplotted are the contours of the JED temperature and optical depth for an accretion rate of 1-2\% Edd., typical of Cyg X-1. The bottom-left panel of Fig. (2) shows the terminal jet velocity $V_{\infty} / c$ as function of the disc ejection efficiency $\xi$ which are in good agreement with observations.

The accretion and ejection properties of JEDs agree with the observations of the prototypical black hole binary Cygnus X-1. The JED solutions are likely to be relevant to the whole class of microquasars.

\section{References}

Blandford, R. D. \& Payne, D. G. 1982, MNRAS, 199, 883

Ferreira, J. \& Pelletier, G. 1993, A\&A, 276, 625+

Malzac, J., Belmont, R., \& Fabian, A. C. 2009, MNRAS, 400, 1512

Petrucci, P.-O., Ferreira, J., Henri, G., Malzac, J., \& Foellmi, C. 2010, A\&A, in press (arXiv:1007.1478) 